\title{
Vibrio vulnificus Vulnibactin, But Not Metalloprotease VvpE, Is Essentially Required for Iron-Uptake from Human Holotransferrin
}

\author{
Choon-Mee KIM,${ }^{a}$ Ra-Young PARK, ${ }^{a}$ Jeong-Hee PARK, ${ }^{a}$ Hui-Yu Sun, ${ }^{a}$ Young-Hoon BAI, ${ }^{b}$ \\ Phil-Yeol Ryu, ${ }^{c}$ Soo-Young KIM, ${ }^{c}$ Joon-Haeng RHEE, ${ }^{c}$ and Sung-Heui SHIN ${ }^{*}, a$ \\ ${ }^{a}$ Research Center for Resistant Cells, ${ }^{b}$ Department of Biology, Chosun University Medical School; Gwangju 501-759: and \\ ${ }^{c}$ Clinical Vaccine R\&D Center, National Research Laboratory of Molecular Microbial Pathogenesis, Research Institute of \\ Vibrio Infection and Genome Research Center for Enteropathogenic Bacteria, and Department of Microbiology, Chonnam \\ National University Medical School; Gwangju 501-746, South Korea.
}

Received October 31, 2005; accepted January 10, 2006

\begin{abstract}
The roles of metalloprotease ( $\mathrm{VvpE})$ and catechol-siderophore (vulnibactin) in the uptake of iron from human transferrins by Vibrio vulnificus have been determined using different experimental conditions and methods. Therefore, in this study, we attempted to elucidate the roles of VvpE and vulnibactin using the same methods and experimental conditions, in an in vitro and a human ex vivo system, and in accordance with the molecular version of Koch's postulates. Neither $v v p E$ mutation nor in trans $v v p E$ complementation affected vulnibactin production, iron-assimilation from human holotransferrin (HT), and bacterial growth in a HT-containing deferrated Heart-Infusion medium (HT-DF-HI) or a HT-containing cirrhotic ascites (HT-CA). In contrast, the mutation of fur gene encoding Fur, a repressor regulating expression of the vulnibactin-mediated iron-uptake system, derepressed vulnibactin production, and facilitated iron-assimilation from HT and bacterial growth in HT-DFHI or HT-CA. The mutation of vis gene encoding isochorismate synthase required for vulnibactin synthesis abolished vulnibactin production, iron-assimilation from HT and bacterial growth in HT-DF-HI or HT-CA. These results demonstrate that vulnibactin is essentially required for iron-assimilation from transferrin, and that $\mathrm{VvpE}$ has no direct effect on facilitating vulnibactin-mediated iron-assimilation from transferrin in vitro or in a human ex vivo system.
\end{abstract}

Key words Vibrio vulnificus; metalloprotease; vulnibactin; transferrin; iron

Vibrio vulnificus is a gram-negative halophilic estuarine bacterium. The pathogen causes fatal and rapidly-progressing septicemia with high mortality. This $V$. vulnificus septicemia (VVS) is closely associated with the consumption of raw seafood contaminated with the bacterium in patients with underlying hepatic disease, heavy alcohol-drinking habits, or other immunocompromised conditions. Several putative virulence factors including capsule, hemolysin, protease, and iron-assimilation systems have been suggested to be associated with VVS, but only capsule and iron-assimilation systems have been confirmed to be authentic virulence factors, in accordance with the molecular version of Koch's postulates. ${ }^{1-3)}$

It has also been well documented that iron and iron-assimilation systems play a crucial role in the pathogenesis of $\mathrm{VVS}^{4-8)}$ VVS pathogenesis is promoted by the elevated serum iron levels, and crucially requires the assimilation of iron from transferrins by $V$. vulnificus. ${ }^{9)} \mathrm{V}$. vulnificus is known to produce two types of siderophores: catechol- and hydroxamate-siderophores. ${ }^{10)}$ Of these, the catechol-siderophore (called vulnibactin) is known to play the more important role in iron-uptake from transferrins and the virulence of $V$. vulnificus. ${ }^{10-14)}$ Expression of the vulnibactin-mediated iron-uptake system is regulated by Fur, a repressor regulating iron uptake. Both the mutation of venB gene encoding an enzyme required for vulnibactin synthesis and the mutation of vuuA gene encoding vulnibactin receptor protein can abolish the ability of $V$. vulnificus to assimilate iron from transferrins and to grow on holotransferrin (HT).

In addition, a metalloprotease (named $\mathrm{VvpE}$ ) of $V$. vulnificus has been extensively studied, and is known to exert a variety of biological effects. ${ }^{1-3,15,16)}$ However, the role of $\mathrm{VvpE}$ in the pathogenesis of VVS remains unclear, as VvpE-deficient mutants showed comparable or higher virulence than wild type strains in mouse experimental models. ${ }^{17-19)}$ Of the various biological activities of $\mathrm{VvpE}$, its role in facilitating $V$. vulnificus iron-uptake via the proteolytic cleavage of heme proteins, transferrins, and lactoferrins has attracted some attention. ${ }^{15,16)}$ However, our previous study demonstrated that an insertional mutation of $v v p E$ gene has no direct effect on iron-assimilation from human transferrins. ${ }^{20)}$

Bacterial iron-uptake systems are themselves virulence factors in many bacterial pathogens, and are promising vaccine targets. $^{21)}$ Also, iron-chelation is considered a prospective therapeutic means of preventing in vivo bacterial growth. ${ }^{22}$ In these regards, it is of considerable importance that bacterial iron-uptake mechanisms be evaluated. However, the roles of vulnibactin and $\mathrm{VvpE}$ in $V$. vulnificus ironuptake from transferrins have been determined using different methods under different experimental conditions. ${ }^{11-13,20)}$ Therefore, we considered that a definitive comparison and reevaluation of the roles of $\mathrm{VvpE}$ and vulnibactin is required, i.e., using the same methods and experimental conditions, in vitro and in a human ex vivo experimental system, and in accordance with the molecular version of Koch's postulates. ${ }^{1)}$ To achieve these ends, we constructed a $V$. vulnificus vvpEdeletion mutant and its $v v p E$-complemented strain, and a furdeletion mutant exhibiting enhanced vulnibactin productivity and a vis-insertion mutant incapable of producing vulnibactin. Using these strains and the methods and conditions established in our previous study, ${ }^{20)}$ we attempted to determine the roles of $\mathrm{VvpE}$ and vulnibactin in iron-uptake from transferrins. 


\section{MATERIALS AND METHODS}

Media, Cirrhotic Ascites, Reagents, Bacterial Strains, Plasmids, and Primers Heart Infusion (designated HI or normal (NL)-HI, BD) broth containing an additional 2\% $\mathrm{NaCl}$ was used as the basal medium for all experiments in this study. NL-HI broth was deferrated using 8-hydroxyquinoline using the method described by Leong and Neilands. ${ }^{23)}$ In brief, NL-HI broth was mixed with an equal volume of 3\% (w/v) solution of 8-hydroxyquinoline, which was dissolved in chloroform, and vigorously stirred for $2 \mathrm{~d}$. The mixture was allowed to stand at room temperature to be separated into aqueous and chloroform layers. The aqueous extracts were mixed and stirred with pure chloroform for over $2 \mathrm{~h}$ in order to remove residual 8-hydroxyquinoline. The residual iron concentration of the deferrated HI (DF-HI) broth was $1.0 \mu \mathrm{g} / \mathrm{dl}$ or less (Diagnostic Iron and Total IronBinding Capacity kit, Sigma-Aldrich). Cirrhotic ascites (CA) were obtained from five patients with liver cirrhosis. Equal volumes of the five CA samples were mixed, and this pooled CA was sterilized and free of human cells using disposable syringe filters $(0.45 \mu \mathrm{m}$, Sartorius), and then incubated at $65^{\circ} \mathrm{C}$ for $30 \mathrm{~min}$ to inactivate possible bactericidal factors including complement. When required, $0.5 \mathrm{mg} / \mathrm{ml}$ of human holotransferrin (HT; $1200-1600 \mu \mathrm{g}$ of iron per $1 \mathrm{~g}$ of pro- tein; Sigma-Aldrich) was added to the DF-HI broth or CA. The concentrations of transferrin and iron are below or within the normal physiological ranges $(2.2$ to $4.0 \mathrm{mg} / \mathrm{ml}$ for transferrin and 50 to $160 \mu \mathrm{g} / \mathrm{dl}$ for iron). In order to select and maintain mutant strains, HI agar and Thiosulfate-CitrateBile Salt Sucrose (TCBS, BD) agar containing appropriate antibiotics; ampicillin $(20 \mu \mathrm{g} / \mathrm{ml})$, kanamycin $(300 \mu \mathrm{g} / \mathrm{ml})$, tetracycline $(2 \mu \mathrm{g} / \mathrm{ml})$ and chloramphenicol $(2 \mu \mathrm{g} / \mathrm{ml})$, were used. Unless noted otherwise, all reagents were purchased from Sigma-Aldrich. The bacterial strains, plasmids, and primers used in this study are listed in Table 1.

Construction of the vvpE-Deletion Mutant and an in trans vvpE-Complemented Strain A $V$. vulnificus vvpEdeletion mutant (CMM1049) was constructed by allelic exchange using R6K origin suicide vector pCVD442, as described in our previous study. ${ }^{24)}$ To restore the $v v p E$ wild type gene in the vvpE-deletional mutant CMM1049 strain, a 2.64$\mathrm{kb}$ BamHI-EcoRI fragment containing 1830-bp $v v p E$ gene was amplified using the PCR primers, Comp-1 with a BamHI overhang and Comp-2 with an EcoRI overhang, and subsequently subcloned into the broad host range vector, pLAFR3II (named pCMM1548), which had been prepared by subcloning a $1453-b p$ Bam HI- $B g / I$ I fragment containing the bla gene of pUTKm1 into a BglII site of pLAFR3. ${ }^{25,26)}$ The pCMM1548 was transferred into the CMM1049 strain

Table 1. Bacterial Strains, Plasmids, and Primers Used in This Study

\begin{tabular}{|c|c|c|}
\hline Strains, plasmids and primers & Characteristics and sequences & Source or reference \\
\hline \multicolumn{3}{|l|}{ V. vulnificus } \\
\hline MO6-24/O & Wild type strain & J. G. Morris, Jr. \\
\hline CMM1049 & MO6-24/O with $v v p E$-deletion mutation & 24) \\
\hline CMM1502 & CMM1049 complemented by wild type $v v p E$ & This study \\
\hline CMM2301 & MO6-24/O with vis-insertion mutation & This study \\
\hline CMM2303 & MO6-24/O with fur-deletion mutation & This study \\
\hline \multicolumn{3}{|c|}{ 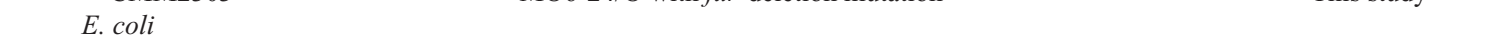 } \\
\hline SY327 $\lambda$ pir & $\begin{array}{l}\Delta(\text { lac pro }) \arg E(\mathrm{Am}) \text { rif nalA recA56 } \lambda \text { pir lysogen; } \\
\text { Host for suicide vector }\end{array}$ & 29) \\
\hline SM10 $\lambda$ pir & $\begin{array}{l}\text { thi thr leu tonA lacY supE recA::RP4-2-Tc } \mathrm{c}^{\mathrm{r}}:: \mathrm{Mu} \mathrm{Km}^{\mathrm{r}} \\
\lambda \text { pir lysogen: Conjugation donor }\end{array}$ & 29) \\
\hline \multicolumn{3}{|l|}{ Plasmids } \\
\hline $\mathrm{pCR}^{\circledR} 2.1-\mathrm{TOPO}^{\circledR}$ & PCR cloning vector & Invitrogen \\
\hline pNQ705 & Suicide vector with $\mathrm{R} 6 \mathrm{~K}$ origin; $\mathrm{Cm}^{\mathrm{r}}$ & 30) \\
\hline pDM4 & Suicide vector with $\mathrm{R} 6 \mathrm{~K}$ origin; $\mathrm{Cm}^{\mathrm{r}}$ & 28) \\
\hline pCMM230 & $\begin{array}{l}\text { PCR-amplified truncated vis gene }(0.67-\mathrm{kb}) \text { cloned } \\
\text { into pCR }{ }^{\mathbb{R}} 2.1 \text {-TOPO }{ }^{\mathbb{}}\end{array}$ & This study \\
\hline pCMM232 & $\begin{array}{l}\text { XbaI-HindIII fragment of pCMM } 230 \text { cloned } \\
\text { into pNQ705 }\end{array}$ & This study \\
\hline pCMM236 & $\begin{array}{l}\text { 1.8-kb fragment containing an in-frame deletion of } \\
\text { V. vulnificus fur gene cloned into } \mathrm{pCR}^{\mathbb{}} 2.1-\mathrm{TOPO}^{\circledR}\end{array}$ & This study \\
\hline pCMM237 & SacI-XbaI fragment of pCMM236 cloned into pDM4 & This study \\
\hline pCMM1548 & $\begin{array}{l}\text { 2.64-kb BamHI-EcoRI fragment containing } v v p E \\
\text { cloned into pLAFR3II }\end{array}$ & This study \\
\hline pLAFR3II & pLAFR3 with bla inserted at the cos site; $\mathrm{Ap}^{\mathrm{r}} \mathrm{Tc}^{\mathrm{r}}$ & This study \\
\hline pLAFR3 & IncP cosmid vector; $\mathrm{Tc}^{\mathrm{r}}$ & 26) \\
\hline pUTKm1 & Tn5-based insertion delivery plasmid, $A p^{r}$ & 25) \\
\hline pRK2013 & IncP, $\mathrm{Km}^{\mathrm{r}}$, Tra $\mathrm{Rk} 2^{+}$repRK2 repE1 & 27) \\
\hline \multicolumn{3}{|l|}{ Primers } \\
\hline Fur-1 & $5^{\prime}$-cgtcggtttatgcattgatgaagttcg-3' & This study \\
\hline Fur-2 & $5^{\prime}$-cgtcgatattacatatactttcccgttgatc- $3^{\prime}$ & This study \\
\hline Fur-3 & $5^{\prime}$-ggaaagtatatgtaatatcgacgetcgatagatc- $3^{\prime}$ & This study \\
\hline Fur-4 & $5^{\prime}$-ctcgacaacatcaccattgattgcc- $3^{\prime}$ & This study \\
\hline Comp-1 & $5^{\prime}$-cgggatccgecatatccccaaatgccgatg- $3^{\prime}$ & This study \\
\hline Comp-2 & $5^{\prime}$-cggaattctcaatattgtagctttaacgtcac- $3^{\prime}$ & This study \\
\hline Vis-1 & $5^{\prime}$-gccaatcaggaactctcg-3' & This study \\
\hline Vis-2 & $5^{\prime}$-ctccagcaaacaccttcatc- $3^{\prime}$ & This study \\
\hline
\end{tabular}


by triparental mating using a conjugative helper plasmid pRK2013. ${ }^{27)}$ The transconjugants were screened on TCBS agar plates containing ampicillin and tetracycline, and the presence of the wild type allele was confirmed by PCR using the primers Comp-1 and Comp-2. The inability of the CMM1049 strain to produce VvpE recovered to the wild type level by the in trans-complementation of wild type $v v p E$ (CMM1502).

Construction of a fur-Deletion Mutant and a vis-Insertion Mutant An in-frame deletion mutant of the $V$. vulnificus fur gene was constructed by crossover PCR. Two pairs of PCR primers (Fur-1/Fur-2 and Fur-3/Fur-4) were used for the PCR amplification of the $V$. vulnificus fur gene. The two PCR products were used as a template for a second PCR amplification using Fur-1 and Fur-4 PCR primers. The resulting PCR product was cloned into $\mathrm{pCR}^{\circledR} 2.1$ - $\mathrm{TOPO}^{\circledR}$ vector (Invitrogen) to generate pCMM236. The $S a c I$ and $X b a I$ fragment was excised from pCMM236 and cloned into a suicide vector pDM4.$^{28)}$ The resulting plasmid pCMM237 was transformed into $E$. coli SY327 $\lambda$ pir and SM10 $\lambda$ pir ${ }^{29)}$ and subsequently transferred to $V$. vulnificus MO6-24/O by conjugation. Transconjugants were selected on TCBS agar containing chloramphenicol, and stable transconjugants were spread onto $\mathrm{HI}$ agar containing $10 \%$ sucrose to allow the second homologous recombination to occur. The resulting mutation in the $V$. vulnificus fur gene (CMM2303) was confirmed by PCR.

Isochorismate synthase (Vis) is an enzyme required for the synthesis of vulnibactin in $V$. vulnificus. Insertional inactivation of the vis gene was achieved using a suicide vector pNQ705. ${ }^{30)}$ A DNA fragment lacking the $5^{\prime}$ and $3^{\prime}$ ends of the ven $B$ gene was amplified by PCR using Vis-1 and Vis-2 primers. The amplified fragment was then cloned into pCR $^{\circledR} 2.1-$ TOPO $^{\circledR}$ vector (pCMM230), and the XbaI-HindIII fragment from pCMM230 was cloned into a suicide vector pNQ705 (pCMM232). The resulting pCMM232 was transformed into $E$. coli SY327 $\lambda$ pir and SM10 $\lambda$ pir ${ }^{29)}$ and transferred to $V$. vulnificus MO6-24/O by conjugation. Stable transconjugants were selected on TCBS agar containing chloramphenicol. The inability of the vis-insertion mutant (CMM2301) to produce vulnibactin was confirmed using the Arnow test. ${ }^{31)}$

Growth Conditions and Monitoring Bacteria were cultured overnight in HI broth containing $100 \mu \mathrm{M}$ dipyridyl in order to adapt the bacteria to iron-restricted conditions and to reduce intracellular iron storage. These preconditioned bacteria in the late exponential growth phase were then inoculated into test media and cirrhotic ascites, and cultured with vigorous shaking $(220 \mathrm{rpm})$ at $37^{\circ} \mathrm{C}$ for $24 \mathrm{~h}$. According to our preliminary experimental results, an initial bacterial inoculum of $1 \times 10^{6} \mathrm{cfu} / \mathrm{ml}$ was used to observe the effect of $\mathrm{VvpE}$, and that of $1 \times 10^{3} \mathrm{cfu} / \mathrm{ml}$ was used to observe the effect of vulnibactin. Bacterial growth was measured by $\mathrm{OD}_{600}$. Culture supernatants were obtained by centrifuging culture aliquots at $10000 \mathrm{rpm}$ for $5 \mathrm{~min}$.

Measurement of Caseinolytic Activity and Zymogram In order to compare the protease production in the $V$. vulnificus strains, we measured the caseinolytic activity and performed zymography. ${ }^{18,20,32)}$ For the measurement of the caseinolytic activity in culture supernatants, a method described previously was used without modification. ${ }^{18,20)}$ For zymography, ${ }^{32)}$ equal volumes $(20 \mu \mathrm{l})$ of culture supernatants were electrophoresed without heat- and mercaptoethanol-denaturation on $12 \%$ SDS-polyacrylamide gel containing $0.3 \%$ Skim milk (BD) or HT. The gels were incubated in re-naturation buffer containing $2.5 \%$ Triton X-100 at room temperature for $1 \mathrm{~h}$ in order to remove SDS, and then in developing buffer containing dithiothreitol and $\mathrm{CaCl}_{2}$ at $4{ }^{\circ} \mathrm{C}$ overnight, and finally stained by Coomassie blue.

Arnow Test and RT-PCR for the vis Gene The production of vulnibactin in culture supernatants was measured using the Arnow test, ${ }^{20,31)}$ and two-fold diluted catechol solutions were used to obtain a reference curve. Total RNA was extracted from the bacterial pellets of the MO6-24/O and CMM2303 strains grown for 6 and $12 \mathrm{~h}$ in DF-HI broth and CA containing $0.5 \mathrm{mg} / \mathrm{ml}$ of HT with easy-Blue ${ }^{\mathrm{TM}}$ reagent (iNtRon Biotechnology, Korea). Two hundred nanograms of total RNA were reverse-transcribed and amplified using OneStep RT-PCR PreMix (iNtRon Biotechnology) and the visspecific primers Vis-1 and Vis-2. The 647-bp PCR product was electrophoresed and stained with ethidium bromide.

SDS-PAGE and 6 м Urea-PAGE SDS-PAGE was used to observe the intactness of $\mathrm{HT}^{20}{ }^{20}$ Culture supernatants $(20 \mu \mathrm{l})$ were mixed with SDS-sample buffer and heated for $5 \mathrm{~min}$, and then electrophoresed on a $6 \%$ stacking and $10 \%$ running gel. $6 \mathrm{M}$ urea-PAGE was used to observe iron-removal from HT. ${ }^{20,33)}$ Culture supernatants $(20 \mu \mathrm{l})$ were mixed with urea-gel sample buffer, and electrophoresed without heating on a $5 \%$ stacking and $6 \%$ running urea gel. Gels were stained with Coomassie blue.

\section{RESULTS}

VvpE Is a Major Protease Capable of Degrading Human Transferrins More profound proteases were produced in NL-HI broth than in DF-HI broth (Figs. 1A, B). Accordingly, in order to confirm the $v v p E$ mutation and complementation and observe profiles of proteases, we compared protease production in NL-HI broth using zymogram containing Skim milk or HT (Fig. 1C). No remarkable difference was observed in the growth of the three strains, the wild type MO6-24/O, the $v v p E$-deleted CMM1049, and the $v v p E$-complemented CMM1502 (data not shown). On the zymogram containing Skim milk, the MO6-24/O strain produced one major protease and at least two minor proteases. The CMM1049 strain produced only the minor proteases, and the CMM1502 strain produced the same proteases as the wild type strain. Accordingly, the major protease appeared to be VvpE. In contrast, on the zymogram containing HT, the MO6-24/O and CMM1502 strains showed only a proteolytic band, but the CMM1049 strain did not show any proteolytic band. Only VvpE appeared to exhibit proteolytic activity on the zymogram containing HT. These results indicate that iron is required for efficient protease production, and that $\mathrm{VvpE}$ is a major protease and the only protease capable of destroying HT, and thus the roles of minor proteases do not have to be considered in the context of the present study.

The vvpE Mutation Did Not Affect Growth on HT, Vulnibactin Production, and Iron-Assimilation from HT under Iron-Limited Conditions The growths of all three V. vulnificus strains (MO6-24/O, CMM1049, CMM1502) were stimulated by adding HT to DF-HI broth (Figs. 2A, B). 
However, no remarkable differences were observed in the abilities of the three strains to grow in DF-HI broth containing HT, especially during the exponential growth phase. The $v v p E$ mutation appeared not to affect vulnibactin production. When vulnibactin production was measured in culture supernatants obtained at $12 \mathrm{~h}$ in DF-HI broth containing HT, no

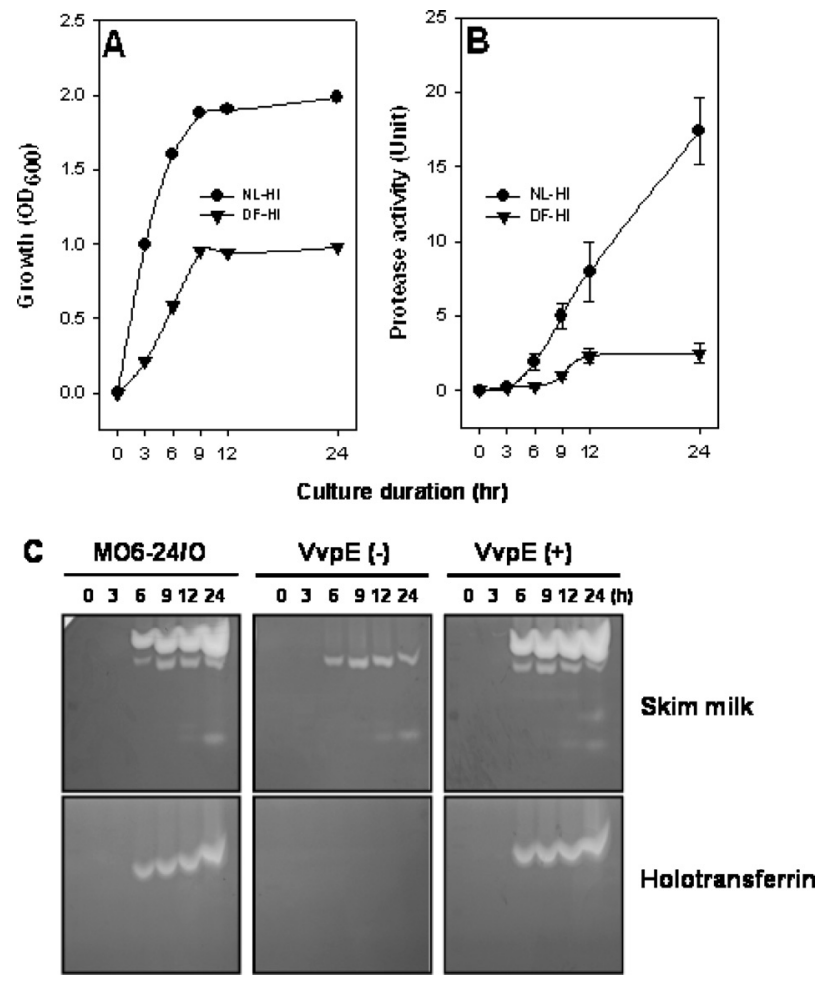

Fig. 1. (A, B) Growth and Protease Production of Vibrio vulnificus

The $V$. vulnificus MO6-24/O strain was cultured with vigorous shaking $(220 \mathrm{rpm})$ in normal (NL)-HI and deferrated (DF)-HI broths at $37^{\circ} \mathrm{C}$ for $24 \mathrm{~h}$. Bacterial growth was measured by $\mathrm{OD}_{600}(\mathrm{~A})$. The caseinolytic activity was measured in culture supernatants obtained at the indicated times (B).

(C) Confirmation of the $v v p E$ Mutation and Complementation on Zymogram

The three $V$. vulnificus strains, MO6-24/O, CMM1049 (VvpE-), CMM1502 $(\mathrm{VvpE}+)$, were cultured with vigorous shaking $(220 \mathrm{rpm})$ in NL-HI broths at $37^{\circ} \mathrm{C}$ for $24 \mathrm{~h}$. Equal volumes $(20 \mu \mathrm{l})$ of culture supernatants obtained at the indicated times were electrophoresed without heat- and mercaptoethanol-denaturation on SDS-polyacrylamide gel containing $0.3 \%$ Skim milk or holotransferrin for zymography. Gels were stained with Coomassie blue. significant difference was observed among the three strains (Fig. 2C). To observe whether or not $V$. vulnificus destroyed HT added to DF-HI broth, SDS-PAGE was performed using equal volumes of culture supernatants (Fig. 3A). None of the three strains destroyed HT, especially during the exponential growth phase. Minor HT destruction was observed during the stationary growth phase, but its extent showed no correlation with the ability of the three strains to grow on HT. To compare the abilities of the three strains to acquire iron from HT, $6 \mathrm{~m}$ urea-PAGE was performed using equal volumes of culture supernatants (Fig. 3B). No remarkable differences were observed among the three strains, and all three strains began to acquire iron from HT during the early exponential growth phase. Similar results were observed when CA was used as a human ex vivo experimental system instead of DFHI broth (Figs. 4A, B). The growths of the three strains were suppressed in CA, but stimulated when HT was added. No

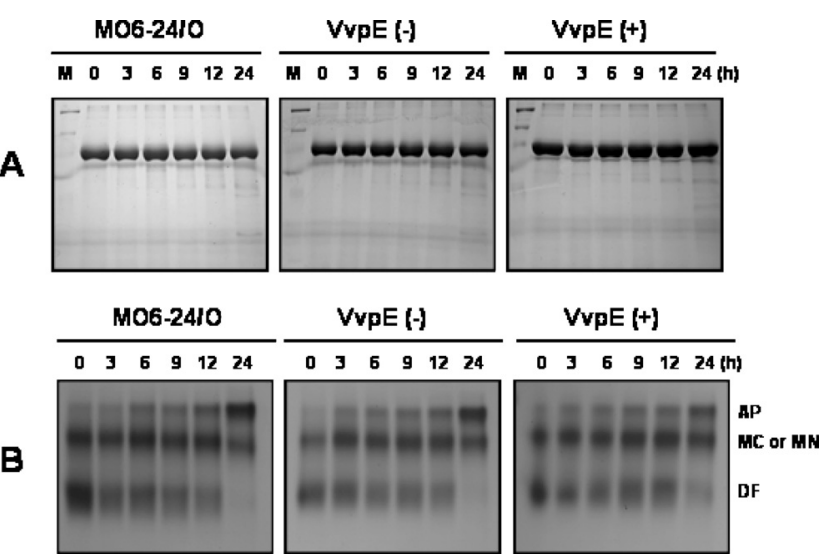

Fig. 3. (A) No Effect of the $v v p E$ Mutation on the Destruction of Holotransferrin (HT)

Bacterial growth is shown in Fig. 2B. Equal volumes ( $20 \mu \mathrm{l})$ of culture supernatants obtained at the indicated times were electrophoresed on SDS-gels, which were then stained with Coomassie blue.

(B) No Effect of the $v v p E$ Mutation on the Acquisition of Iron from HT

Equal volumes $(20 \mu 1)$ of culture supernatants obtained at the indicated times were electrophoresed on $6 \mathrm{~m}$ urea-gels, which were then stained with Coomassie blue. The transferrin is separated into four forms according to iron-saturation level on $6 \mathrm{M}$ ureagel; apoferric (AP), C-terminal monoferric (MC), N-terminal monoferric (MN), and diferric (DF) forms.
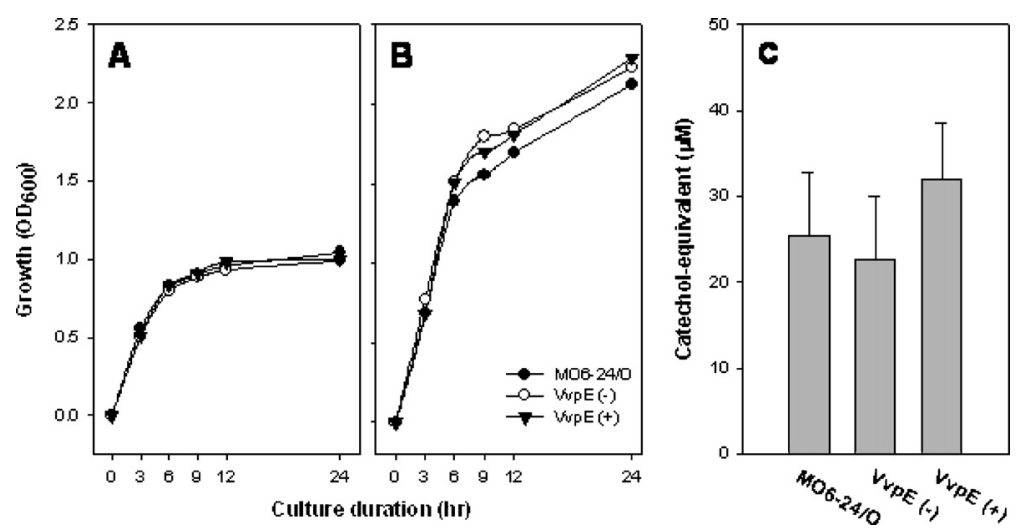

Fig. 2. (A, B) No Effect of the $v v p E$ Mutation on the Growth of $V$. vulnificus on Holotransferrin (HT)

The three $V$. vulnificus strains, MO6-24/O, CMM1049 (VvpE-), CMM1502 (VvpE+), were inoculated into deferrated (DF)-HI broth (A) or DF-HI broth containing 0.5 mg/ml of HT (B) to concentrations of $1 \times 10^{6} \mathrm{cfu} / \mathrm{ml}$, and then cultured with vigorous shaking $(220 \mathrm{rpm})$ at $37^{\circ} \mathrm{C}$ for $24 \mathrm{~h}$. Bacterial growth was measured by $\mathrm{OD}_{600}$ of culture aliquots obtained at the indicated times.

(C) No Effect of the $v v p E$ Mutation on the Production of Vulnibactin

Culture supernatants were obtained from the culture B at $12 \mathrm{~h}$. Vulnibactin production in the culture supernatants was measured by the Arnow test and expressed as catecholequivalent. 


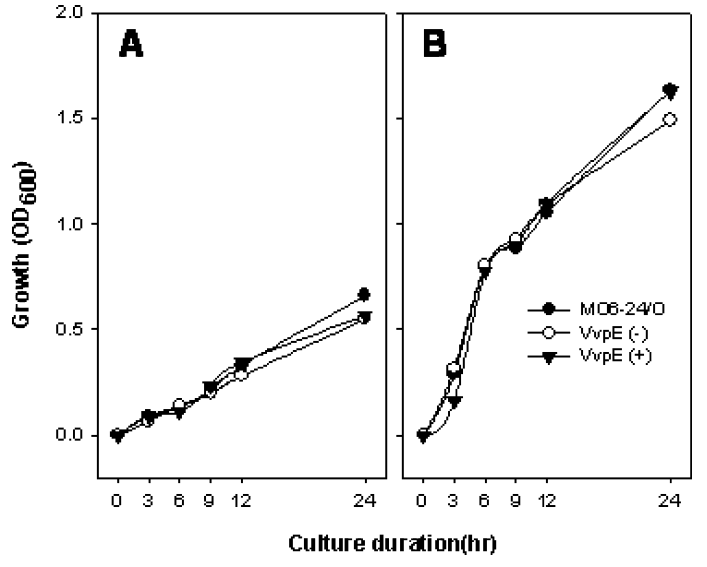

Fig. 4. No Effect of the $v v p E$ Mutation on the Growth of $V$. vulnificus in Cirrhotic Ascites (CA)

The three $V$. vulnificus strains, MO6-24/O, CMM1049 (VvpE-), CMM1502 $(\mathrm{VvpE}+)$, were inoculated in CA (A) or CA containing $0.5 \mathrm{mg} / \mathrm{ml}$ of holotransferrin (B) to concentrations of $1 \times 10^{6} \mathrm{cfu} / \mathrm{ml}$, and then cultured with vigorous shaking $(220 \mathrm{rpm})$ at $37^{\circ} \mathrm{C}$ for $24 \mathrm{~h}$. Bacterial growth was measured by $\mathrm{OD}_{600}$ of culture aliquots obtained at the indicated times.
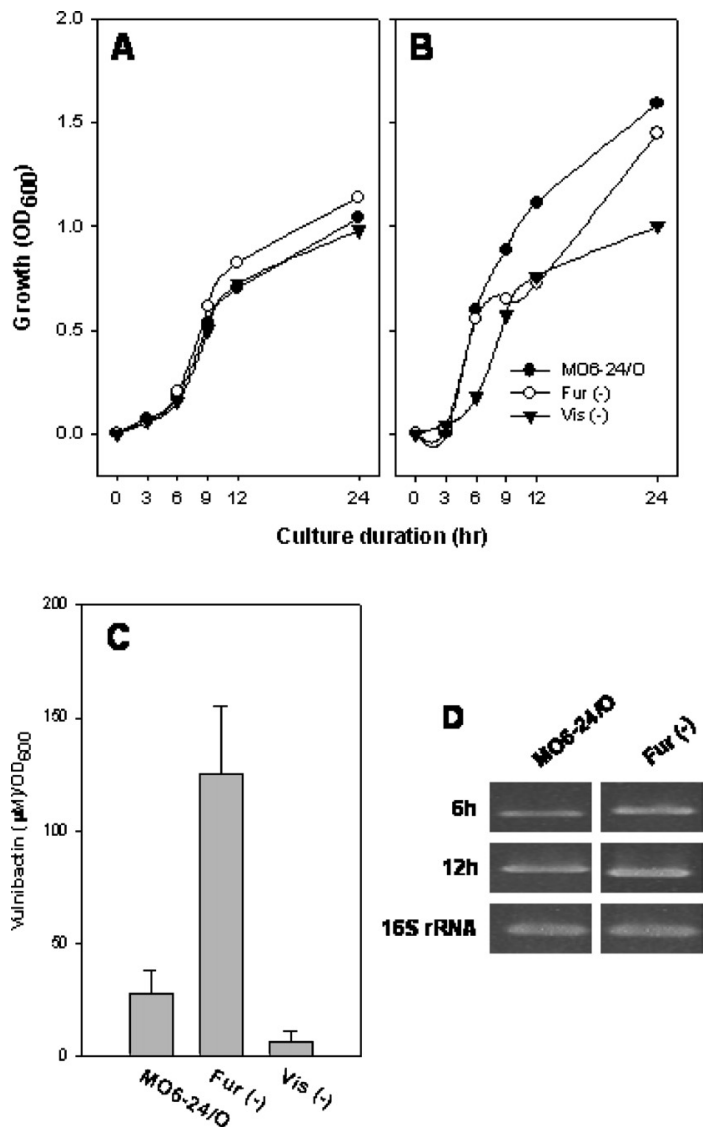

Fig. 5. (A, B) Effect of the fur and vis Mutations on the Growth of $V$. vulnificus on Holotransferrin

The three $V$. vulnificus strains, MO6-24/O, CMM2303 (Fur-), CMM2301 (Vis-), were inoculated into DF-HI broth (A) or DF-HI containing $0.5 \mathrm{mg} / \mathrm{ml}$ holotransferrin (B) to concentrations of $1 \times 10^{3} \mathrm{cfu} / \mathrm{ml}$, and then cultured with vigorous shaking $(220 \mathrm{rpm})$ at $37^{\circ} \mathrm{C}$ for $24 \mathrm{~h}$. Bacterial growth was measured by $\mathrm{OD}_{600}$ of culture aliquots obtained at the indicated times.

(C) Effect of the fur and vis Mutations on the Production of Vulnibactin

Culture supernatants were obtained from culture B at $12 \mathrm{~h}$, and vulnibactin production in the culture supernatants was measured by the Arnow test and expressed as catechol-equivalent.

(D) Effect of the fur Mutation on the vis Transcription

Bacterial pellets were obtained from culture B at 6 and $12 \mathrm{~h}$, total RNA was isolated from each pellet, and RT-PCR was performed using the Vis- 1 and Vis-2 primers.

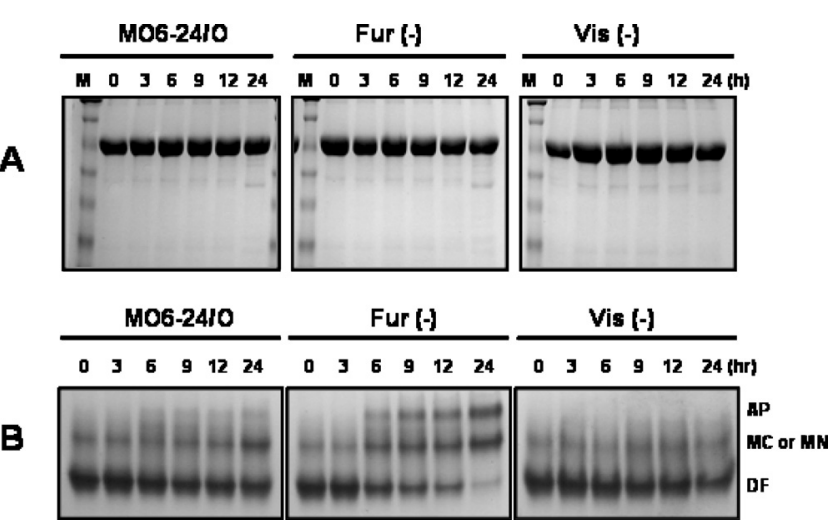

Fig. 6. (A) No Effect of the fur and vis Mutations on the Destruction of Holotransferrin (HT)

Bacterial growth is shown in Fig. 5B. Equal volumes $(20 \mu \mathrm{l})$ of culture supernatants obtained at the indicated times were electrophoresed on SDS-gels, which were stained with Coomassie blue.

(B) Effect of the fur and vis Mutations on the Acquisition of Iron from HT

Equal volumes $(20 \mu \mathrm{l})$ of culture supernatants obtained at the indicated times were electrophoresed on $6 \mathrm{~m}$ urea-gels, which were stained with Coomassie blue. The transferrin is separated into four forms according to iron-saturation level on $6 \mathrm{~m}$ urea-gel apoferric (AP), C-terminal monoferric (MC), N-terminal monoferric (MN), and diferric (DF) forms.

noticeable difference was observed among the three strains in terms of their abilities to grow on HT in the CA background. The above results indicate that, even though it may be produced, $\mathrm{VvpE}$ does not facilitate the iron-assimilation and growth of $V$. vulnificus on HT, especially when HT is supplied as the sole iron-source to an iron-limited environment.

Vulnibactin Is Essentially Required for Growth on HT and for Iron-Assimilation from HT under Iron-Limited Conditions In order to confirm that the ability of $V$. vulnificus to utilize HT was dependent on its ability to produce vulnibactin, we used the fur-deletion mutant CMM2303 strain and the vis-insertion mutant CMM2301 strain. The growth of the wild type MO6-24/O strain was stimulated when HT was added into DF-HI broth (Figs. 5A, B). The growth of the CMM2303 strain also appeared to be stimulated by HT, though it was abruptly impaired during the late exponential growth phase (Fig. 5B). This growth characteristic of the CMM2303 strain was repeatedly observed during repeated experiments, especially in the late exponential and early stationary growth phases, and under iron-rich conditions. However, no further characterization of the phenotype of the CMM2303 strain was conducted because Fur is a wellknown global regulator, and thus a fur mutation may affect the expressions of many phenotypes. In contrast, the growth of the CMM2301 strain was not stimulated by adding HT to DF-HI broth (Figs. 5A, B).

Vulnibactin production by the three strains was compared in culture supernatants obtained after $12 \mathrm{~h}$ of culture in DFHI broth containing HT (Fig. 5C). It was found that the CMM2303 strain produced a greater amount of vulnibactin, despite its growth impairment, than the wild-type strain. On the other hand, the CMM2301 strain did not produce vulnibactin in the medium. According to RT-PCR, the transcriptional levels of the vis gene in the MO6-24/O and CMM2303 strains were consistent with their vulnibactin productions (Fig. 5D). On SDS-PAGE (Fig. 6A), none of the three strains destroyed HT. However, on $6 \mathrm{M}$ urea-PAGE (Fig. 6B), the CMM2303 strain was able to assimilate iron from HT more 

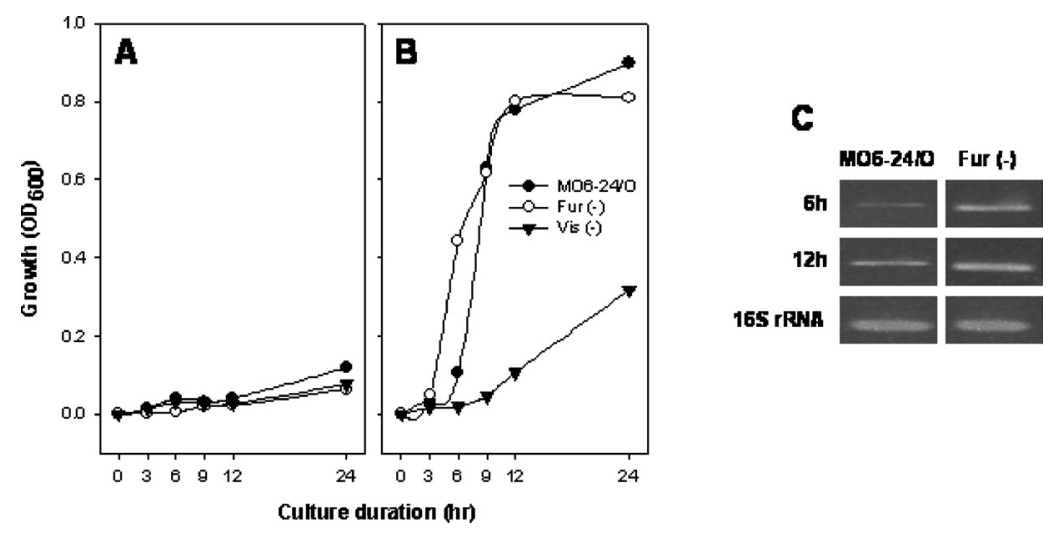

Fig. 7. Effect of the fur and vis Mutations on the Growth of $V$ vulnificus in Cirrhotic Ascites (CA)

The three $V$. vulnificus strains, MO6-24/O, CMM2303 (Fur-), CMM2301 (Vis-), inoculated in CA (A) or CA containing $0.5 \mathrm{mg} / \mathrm{ml}$ of holotransferrin (B) to concentrations of $1 \times 10^{3} \mathrm{cfu} / \mathrm{ml}$, and then cultured with vigorous shaking at $37^{\circ} \mathrm{C}$ for $24 \mathrm{~h}$. Bacterial growth was measured by $\mathrm{OD}_{600}$ of culture aliquots obtained at the indicated times. (C) The vis transcription in a CA background. Bacterial pellets were obtained from culture B at 6 and $12 \mathrm{~h}$, total RNA was isolated from each pellet, and RT-PCR was performed using the Vis1 and Vis-2 primers.

efficiently than the wild type strain. In contrast, the CMM2301 strain was unable to assimilate iron from HT. Similar results were also observed when CA was used as a human ex vivo experimental system instead of DF-HI broth (Figs. 7A,B). The growths of the three strains were suppressed in $\mathrm{CA}$, and the growths of the MO6-24/O and CMM2303 strains were stimulated by addition of HT to CA. The CMM2303 strain began to grow earlier in CA containing HT than the wild type strain. In contrast, the growth of the CMM2301 strain remained suppressed despite the addition of HT. The above results indicate that the ability of $V$. vulnificus to grow on HT is dependent on its ability to produce vulnibactin, and thus to acquire iron from HT.

\section{DISCUSSION}

A research group suggested that VvpE could facilitate iron-assimilation by $V$. vulnificus via the proteolytic cleavage of iron-withholding host proteins such as transferrins. ${ }^{15,16)}$ However, in this study, purified VvpE was exogenously added to a culture medium in order to induce the proteolytic cleavage of transferrin or lactoferrin at culture initiation, which does not accurately simulate VvpE production during batch culture. In our previous study, ${ }^{20)}$ we argued that $\mathrm{VvpE}$ plays a role in iron-uptake from HT. In brief, (1) no difference was observed between the abilities of a $v v p E$-insertion mutant and its wild type strain to grow in DF-HI broth containing HT, (2) both $v v p E$ transcription and extracellular $\mathrm{VvpE}$ production occurred in the late growth phase after $V$. vulnificus had already consumed most of the iron present for growth, (3) moreover, only a small amount of VvpE was produced and HT was not destroyed in DF-HI broth containing HT, and (4) no difference was observed in the abilities of the $v v p E$-insertion mutant and its wild type strain to acquire iron from HT. However, our previous study did not fulfill the molecular version of Koch's postulates ${ }^{1)}$ because only a $v v p E$-insertion mutant was used.

V. vulnificus vulnibactin is known to play important roles in the iron-uptake from transferrins and the expression of virulence. ${ }^{10-14)}$ Litwin and co-workers reported that a ven $B \mathrm{mu}-$ tant incapable of producing vulnibactin could not acquire iron from transferrins, and thus could not grow on agars con- taining transferrins. In addition, they showed that a vuuA mutant incapable of expressing vulnibactin receptor could also neither acquire iron from transferrins nor grow on the agars containing them. ${ }^{11,13)}$ However, they did not rule out the effect of $\mathrm{VvpE}$ on the vulnibactin-mediated iron-uptake from transferrin.

As described above, the roles of Vibrio vulnificus VvpE and vulnibactin in iron-uptake from transferrins have been investigated by different research groups using different experimental conditions and methods. Bacterial iron-uptake systems are considered to be virulence factors in many bacterial pathogens, and thus to be promising targets for the development of vaccines and new chemotherapeutic agents. ${ }^{21,22)}$ Therefore, the elucidation of bacterial iron-uptake mechanisms is of some importance. In the present study, the roles of $\mathrm{VvpE}$ and vulnibactin were examined using the same methods and the same experimental conditions, in vitro and in a human ex vivo experimental system, and moreover, in accordance with the molecular version of Koch's postulates. ${ }^{1)}$

The $v v p E$-deletion mutant CMM1049 strain grew as robustly as the wild type MO6-24/O and $v v p E$-complemented CMM1502 strains when HT was supplemented as the sole iron source to DF-HI broth (Fig. 2B) or CA (Fig. 4B). Moreover, none of the three strains destroyed HT added to DF-HI broth (Fig. 3B), although both the MO6-24/O and CMM1502 strains had the ability to produce VvpE capable of destroying HT (Fig. 1C). A minute HT destruction observed during the stationary growth phase (Fig. 3A) is believed to have been the result of the nonspecific destruction of HT because it was also observed in the $v v p E$-deletion mutant CMM1049 strain (Fig. 3A), and $\mathrm{VvpE}$ was the only protease capable of destroying HT (Fig. 1C).

Most iron is required for exponentially growing $V$. vulnificus cells, whereas VvpE seems to act when cells have already grown. In our previous study, ${ }^{20)} \mathrm{VvpE}$ expression was observed only in the late exponential or stationary growth phases when most iron had already been consumed. Without exception, reports issued to date demonstrate that VvpE expression occurs during the late exponential or stationary growth phase. ${ }^{34-37)}$ These results evidently indicate that VvpE is not involved in facilitating vulnibactin-mediated iron-assimilation via the proteolytic cleavage of transferrins. 
DF-HI broth containing HT as an iron source and human body fluids present an iron-limited condition because transferrin-bound iron is not freely available for $V$. vulnificus. Therefore, in order for VvpE to facilitate iron-uptake via the proteolytic cleavage of transferrins, $\mathrm{VvpE}$ must be produced profoundly in iron-limited conditions. However, VvpE appeared to be produced more profoundly in iron-rich conditions than in iron-limited conditions, as shown in Fig. 1B. Shinoda and co-workers recently reported that a high growth rate of $V$. vulnificus in conditions with an abundance of available iron is essential for the efficient production of proteases. ${ }^{38,39)}$ These results indicate that iron is required for efficient $\mathrm{VvpE}$ production, and that $\mathrm{VvpE}$ is not required for efficient iron-uptake. However, controversy remains between VvpE production and iron concentration. In our previous study, ${ }^{20)}$ we found that $v v p E$ transcription was stimulated, but extracellular VvpE production was not stimulated by adding $\mathrm{HT}$ or $\mathrm{FeCl}_{3}$ to DF-HI. This discrepancy indicates that undefined posttranscriptional events might be involved in the extracellular VvpE production. Accordingly, further studies about these posttranscriptional processes are necessary.

Our results show no difference among the abilities of the three strains, the MO6-24/O, CMM1049, and CMM1502 strains, to grow in DF-HI broth containing HT, which appears to be attributed to their similar levels of vulnibactin production (Fig. 2C). Since the CMM1049 strain was found to assimilate iron from HT as efficiently as the other two strains (Fig. 3B), vulnibactin appears to be able to uptake iron without the assistance of the VvpE-mediated proteolytic cleavage of HT. These results were reinforced by the findings obtained for the fur-deletion mutant CMM2303 strain and the vis-insertion mutant CMM2301 strain. The CMM2303 strain produced a greater amount of vulnibactin (Fig. 5C) and assimilated iron from HT more efficiently than the wild type strain (Fig. 6B). In contrast, the CMM2301 strain was unable to produce vulnibactin (Fig. 5C) or to uptake iron from HT (Fig. 6B). Moreover, the growth of the CMM2303 strain was stimulated in DF-HI and CA by adding HT to a level greater than the wild type strain, but the growth of the CMM2301 strain remained suppressed in both DF-HI broth and CA in spite of the addition of HT (Figs. 5, 7). Moreover, no noticeable difference was observed in the abilities of the two strains (CMM2301, CMM2303) to produce VvpE (data not shown), and all three strains failed to destroy HT added to DF-HI broth (Fig. 6A). Although the methods and conditions used were different from those of the present study, Litwin and co-workers also demonstrated that both a venBmutant and a vuuA-deficient mutant were unable to grow on transferrin-bound iron. ${ }^{11-13)}$ The above results indicate that vulnibactin is essentially required for iron-uptake from transferrins, and that $\mathrm{VvpE}$ has no direct effect on facilitating vulnibactin-mediated iron-uptake from transferrins both in vitro and in a human ex vivo experimental system.

In conclusion, this study demonstrates that the production of vulnibactin is essentially required for iron-assimilation from transferrins by $V$. vulnificus, and that $\mathrm{VvpE}$ is not involved in facilitating this vulnibactin-mediated iron-uptake from HT both in vitro and in a human ex vivo system. Therefore, these results suggest that the vulnibactin-mediated ironuptake system may be an attractive target for vaccine development for the prevention of VVS.
Acknowledgements This study was supported by a grant (no. R13-2003-009) from the Korean Science and Engineering Foundation through the Research Center for Resistant Cells, and by the research fund (2005) of Chosun University. We thank Dr. Haeng-Jong Song for his helpful discussion.

\section{REFERENCES}

1) Falkow S., Rev. Infect. Dis., 10, S274-S276 (1988).

2) Strom M. S., Paranjpye R. N., Microb. Infect., 2, 177-188 (2000).

3) Gulig P. A., Bourdage K. L., Starks M. A., J. Microbiol., 43, 118-131 (2005).

4) Hor L. I., Chang T. T., Wang S. T., J. Infect. Dis., 179, 275-278 (1998).

5) Wright A. C., Simpson L. M., Oliver J. D., Infect. Immun., 34, 503507 (1981).

6) Hor L. I., Chang Y. K., Chang C. C., Lei H. Y., Ou J. T., Microbiol. Immunol., 44, 871-878 (2000).

7) Brennt C. E., Wright A. C., Dutta S. K., Morris J. G., Jr., J. Infect. Dis., 164, 1030-1032 (1991).

8) Starks A. M., Schoeb T. R., Tamplin M. L., Parveen S., Doyle T. J., Bomeisl P. E., Escudero G. M., Gulig P. A., Infect. Immun., 68, 57855793 (2000).

9) Morris J. G., Wright A. C., Simpson L. M., Wood P. K., Johnson D. E., Oliver J. D., FEMS Microbiol. Lett., 40, 55-59 (1987).

10) Simpson L. M., Oliver J. D., Infect. Immun., 41, $644-649$ (1983).

11) Webster A. C. D., Litwin C. M., Infect. Immun., 68, 526-534 (2000).

12) Litwin C. M., Calderwood S. M., J. Bacteriol., 175, 706-715 (1993).

13) Litwin C. M., Rayback T. W., Skinner J., Infect. Immun., 64, 2824 2838 (1996).

14) Shin S. H., Chung S. S., Rhee J. H., J. Bacteriol. Virol., 31, 325-331 (2001).

15) Okujo N., Akiyama T., Miyoshi S., Shinoda S., Yamamoto S., Microbiol. Immunol., 40, 595-598 (1996).

16) Nishina Y., Miyoshi S., Nagase A., Shinoda S., Infect. Immun., 60, 2128 - 2132 (1992).

17) Shao C. P., Hor L. I., Infect. Immun., 68, 3569-3573 (2000).

18) Jeong K. C., Jeong H. S., Rhee J. H., Lee S. E., Chung S. S., Starks A. M., Escudero G. M., Gulig P. A., Choi S. H., Infect. Immun., 68 5096-5106 (2000).

19) Fan J. J., Shao C. P., Ho Y. C., Yu C. K., Hor L. I., Infect. Immun., 69, 5943-5948 (2001).

20) Shin S. H., Sun H. Y., Park R. Y., Kim C. M., Kim S. Y., Rhee J. H., FEMS Microbiol. Lett., 247, 221-229 (2005).

21) Brown J. S., Ogunniyi A. D., Woodrow M. C., Holden D. W., Paton J. C., Infect. Immun., 69, 6702-6706 (2001).

22) Lesic B., Foulon J., Carniel E., Antimicrob. Agents Chemother, 46 $1741-1745$ (2002).

23) Leong S. A., Neilands J. B., Arch. Biochem. Biophys., 218, 351-359 (1982).

24) Shin S. H., Sun H. Y., Choi M. H., Park R. Y., Bai Y. H., Kim C. M., Kim S. Y., Kim Y. R., Lee S. E., Rhee J. H., Biol. Pharm. Bull., 28, $1294-1297$ (2005).

25) Herrero M., de Lorenzo V., Timmis K. N., J. Bacteriol., 172, 6568 6572 (1990).

26) Staskawicz B., Dahlbeck D., Keen K., Napoli C., J. Bacteriol., 169 5789-5794 (1987).

27) Ditta G., Stanfield D. C., Helinski D. R., Proc. Natl. Acad. Sci. U.S.A., 27, 7347-7351 (1980).

28) McGee K., Horstedt P., Milton D. L., J. Bacteriol., 178, 5188—5198 (1996).

29) Miller V., Mekalanos J. J., J. Bacteriol., 170, 2575-2583 (1988).

30) Milton D. L., Norqvist A., Wolf-Watz H., J. Bacteriol., 174, 72357244 (1992).

31) Arnow L. E., J. Biol. Chem., 118, 531-537 (1937).

32) Raser K. J., Posner A., Wang K. K., Arch. Biochem. Biophys., 319, 211-216 (1995).

33) Makey D. G., Seal U. S., Biochem. Biophys. Acta, 453, 250-256 (1976).

34) Hülsmann A., Rosche T. M., Kong I. S., Hassan H. M., Beam D. M., Oliver J. D., Appl. Environ. Microbiol., 69, 6114-6120 (2003). 
35) Jeong H. S., Lee M. H., Lee K. H., Park S. J., Choi S. H., J. Biol. Chem., 278, 45072-45081 (2003).

36) Shao C. P., Hor L. I., J. Bacteriol., 183, 1369-1375 (2001).

37) Kim S. Y., Lee S. E., Kim Y. R., Kim C. M., Ryu P. Y., Choy H. E., Chung S. S., Rhee J. H., Mol. Microbiol., 48, 1647-1664 (2003).
38) Watanabe H., Miyoshi S., Kawase T., Tomochika K., Shinoda S., Microb. Pathogen., 36, 117-123 (2004).

39) Kawase T., Miyoshi S., Sultan Z., Shinoda S., FEMS Microbiol. Lett., 240, 55-59 (2004). 